\title{
Towards an Indoor Level-of-Detail Model for Route Visualization
}

\author{
Benjamin Hagedorn, Matthias Trapp, Tassilo Glander, Jürgen Döllner \\ Hasso-Plattner-Institute \\ University of Potsdam \\ 14482 Potsdam, Germany \\ \{benjamin.hagedorn|matthias.trapp|tassilo.glander|doellner $\} @$ hpi.uni-potsdam.de
}

\begin{abstract}
Indoor routing represents an essential feature required by applications and systems that provide spatial information about complex sites, buildings and infrastructures such as in the case of visitor guidance for trade fairs and customer navigation at airports or train stations. Apart from up-todate, precise $3 D$ spatial models these systems and applications need user interfaces as core system components that allow users to efficiently express navigation goals and to effectively visualize routing information. For interoperable and flexible indoor routing systems, common specifications and standards for indoor structures, objects, and relationships are needed as well as for metadata such as data quality and certainty. In this paper, we introduce a classification of indoor objects and structures taking into account geometry, semantics, and appearance, and propose a level-of-detail model for them that supports the generation of effective indoor route visualization.
\end{abstract}

\section{Introduction}

\subsection{Motivation}

A growing number of applications and systems manage, process, and communicate spatial information about complex sites, buildings and infrastructures based on geospatial 3D models such as virtual 3D city models. Indoor routing represents an important feature that can be implemented on top of these models. It aims at revealing routes to the users, to facilitate understanding of locations and paths, as well as to instruct and guide users to a specific destination within a complex 3D environment.

Examples of applications include visitor guidance for trade fairs, user direction in libraries, and customer navigation at airports or train stations. Typical types of destinations range from rather large entities, such as rooms or elevators, to small entities, such as books in libraries.

Systems and applications for general indoor routing visualization have to manage and process geometry, topology, and thematic data about the underlying spatial 3D models. In particular, they have to integrate $2 \mathrm{D}$ and $3 \mathrm{D}$ data from
GIS, CAD, and BIM into a single information framework and have to ensure up-to-date, precise contents.

Indoor routing, on the one hand, relies on static information about an environment, e.g., geometry models of rooms and furniture, and, on the other hand, has to consider dynamic information, e.g., sensor data, tracking data, or room allocation schedules. Compared to outdoor navigation, indoor routing generally includes more fine-grained features and requires for higher data accuracy.

Indoor routing requires user interfaces that allow users to efficiently express navigation goals and to effectively visualize routing information. Typically, indoor routing visualization takes into account different building structures (e.g., storeys, rooms, walls, doors) and is designed for specific presentation media. Examples for indoor visualization include two-dimensional floor plans and escape route maps. In the case of complex environments such as multistorey buildings, the third dimension complicates the design and automated generation of comprehensible indoor routing visualization. Frequently, manually made three-dimensional cut-away views can be found but cannot be directly generated by today's GIS.

Current indoor routing is faced by manifold limitations and difficulties. Imagine visitors of a trade fair that are searching a specific booth. Typical solutions provide 2D or 3D maps showing a perspective view of the exhibition halls, whereby visitors have to identify their own position, to align their orientation with the map's one, to find the route by themselves, and to memorize key information for their walk to the destination. In addition to the cognitive workload imposed on the visitors, these indoor maps are usually manually designed for each single, specific application case. Thus, they are expensive and cannot take into account dynamic modifications in an environment's structure and usage; the integration of dynamic building information, such as real-time sensor data or tracking data, based on RFID or WLAN, cannot be supported at all.

While base models of geovirtual 3D environments have been standardized by CityGML [5], a data exchange model for virtual 3D city models, as well as by the Industry Foundation Classes (IFC) [4], a neutral, open specification of building information models (BIM), there are less known proposals of level-of-detail models for indoor objects and 
structures. Finally, no formalized model describes so far the classification and integration of detailed indoor building data, routing data, and their visual representation.

In this paper, we present and discuss a classification of indoor objects and structures that takes into account their geometry, appearance, and semantics. Based on this classification we derive an integrated indoor level-of-detail model, which includes thematic, geometric, and routingrelated information. As key application, our concept facilitates the generation of effective indoor route visualization.

\subsection{The Need for Indoor Level-of-Detail}

Geospatial models are generally faced by their inherent complexity. To reduce complexity in a systematic way, e.g., to achieve the right level of abstraction for a specific application or task, level-of-detail (LOD) models are needed and have to be specified for corresponding modeling schemas and formats [10].

Different user tasks lead to different requirements with respect to complexity of indoor models. Similarly, different visualization techniques (e.g., mobile mapping versus interactive desktop applications) exhibit different constraints on data complexity. In particular, for building and site models, semantics, geometry and appearance data has to be provided at various LODs to cope with massive data and limited resources (e.g., storage space, transmission bandwidth, onboard memory). Furthermore, systems and applications can take advantage of LOD indoor data to adapt processing and visualization to specific output media or communication devices. Therefore, indoor data specification, exchange, and usage all require a sound definition of different complexity levels.

To support interoperable and flexible indoor routing visualization in summary, an LOD concept is needed. It helps to define standards for mandatory and optional indoor structures, objects, relationships, data quality, and visual appearance. While CityGML describes LODs for city models, there are no similar approaches for building interiors.

This paper is structured as follows. Section 2 reviews existing approaches for indoor routing and visualization. Section 3 describes a novel concept and model for indoor LOD. Section 4 concludes this paper and outlines directions of future research.

\section{Related Work}

\subsection{Route Finding}

In general, route finding is mainly based on graph structures, i.e., nodes and edges, representing, e.g., relevant locations and routes. A route can be retrieved from such graphs by applying graph search algorithms such as Dijkstra or AStar [16], or knowledge-based approaches [8].

In addition to geometrical and topological data, usercentered and domain-specific indoor navigation requires navigation semantics of indoor structures and navigation context information, which can be provided, e.g., by navigation ontologies [19] and hierarchical models for building topology [3] [9] [18]. In [6], a dual graph for representing topological relationships among indoor entities such as rooms and corridors is introduced. In [1], rooms are decomposed into smaller parts for the integration of sensor data for route finding and navigation.

\subsection{Indoor Building Models}

For effective indoor routing and visualization, appropriate models and data sources are required. At least, they have to represent building-relevant entities (e.g., rooms and doors), their geometry, as well as topological data.

In the domain of building architecture, engineering, construction, and maintenance (AECM), so called building information models (BIM) provide such data. Considering the whole building life cycle, as well as related stakeholders, they aim at interoperability between participating systems and applications. This requires common information models and data formats such as provided by IFC and CityGML, which serve as two standards for the specification of building-relevant structures and related geometries.

Additionally, indoor building models need to represent application-specific information (object types and attributes); e.g., in a fire scenario it is crucial to know about hazardous materials in a building. For that, IFC provides generic PropertySets and domain extensions; CityGML is also easily extendible by generic attributes and application domain extensions (ADEs).

For indoor navigation, routing graphs either have to be represented explicitly as part of the building model or have to be derived from the building entities' geometry and their topological and spatial relationships. A BIM can be considered as source for the extraction of routing-relevant building information [7] [17]. IFC provides a topology resource, which could be used for specifying a routing network by edges, which are attributed by geometry and start and end vertices. CityGML does not support indoor routing; however, the network representation of its TransportationComplex could be used, or an appropriate ADE could be specified.

\subsection{Indoor Routing Visualization}

To provide effective indoor routing visualization, information about the application context and users should be considered, such as application domains and application purposes as well as user needs and abilities. For example, rescue plans must conform to common style guides (e.g., 

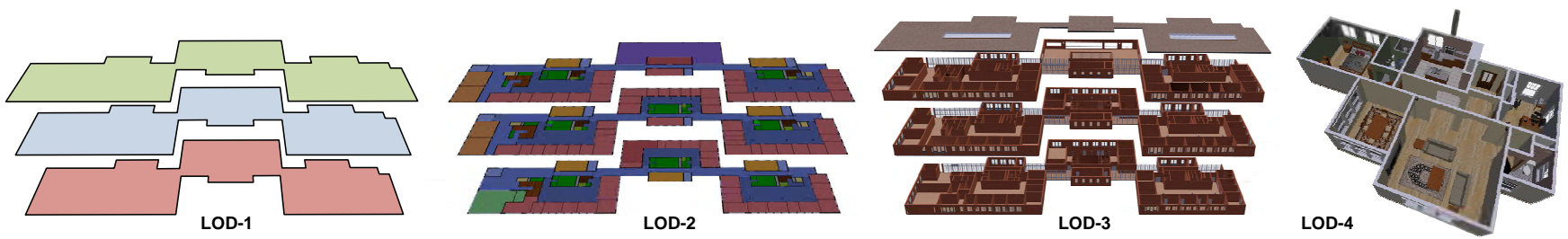

Figure 1. Indoor model instances at different LODs.

DIN 4844-3); they have to include only relevant information and structures (e.g., the position of the viewer, escape routes, fire extinguishers) and should rely on distinguishable colors and well understandable symbols.

Further on, indoor routing visualization depends on properties of the presentation devices such as large or small displays, head-mounted displays, or classic paper. They provide different screen size, screen resolution, and flexibility (static vs. dynamic visualization); they vary in costs as well as provide different degrees of privacy protection.

To support users in navigation and orientation when following a route, additional landmarks should be used along the route [2]. In [11], relevant landmarks supporting indoor evacuation are discussed, and an indoor landmark taxonomy and visualization concept for mobile displays is presented. The annotation of indoor visualization could provide additional support for orientation and navigation [21].

3D visualization could provide a better insight into the spatial configuration and the vertical structuring of a building; the inclusion of well-known in-house elements like furniture objects helps providing a scale [13] [20].

\section{Towards an Indoor Level-of-Detail Model}

Different application scenarios have a range of different requirements and therefore need appropriate representations of a building. For routing within buildings, we propose four relevant indoor LODs, LOD-1 to LOD-4, which are different in their thematic, geometric, topological, and visual complexity. On a technical level, they differ in data size and rendering complexity; on a cognitive level, they provide different degrees of spatial awareness and navigation support. Fig. 1 shows exemplary instances of a building of a low architectural complexity. In the context of indoor navigation, the four LODs support the following use cases:

- LOD-1: Showing a building's access points, showing a building's outer shell, and showing a building's building parts.

- LOD-2: Showing various spaces, showing indoor routes, and providing 2D floor plans.

- LOD-3: Showing space height, showing the shape of doors and windows, and providing higher realism.

- LOD-4: Showing all interior and exterior details of buildings, providing highest degree of realism.

\subsection{Indoor Building Model}

Our proposed indoor LOD building model consists of three components: thematic model, geometry model, and routing model. Fig. 2 illustrates the linkage between thematic model and routing model.

Thematic Model: The abstract type BuildingObject serves as base for each object type of the thematic model; it provides common attributes, such as object identifier and name. This basic structure is designed in analogy to the class concepts of CityGML and IFC.

Buildings and BuildingParts, which are both of type AbstractBuilding, represent the coarsest thematic entities of the model. A Building can comprise several BuildingParts, which represent vertical partitions. Both can contain multiple Floors, which represent horizontal partitions. Floors can group Spaces, such as rooms, halls, staircases, etc. They are bounded by Walls, which can contain Openings (e.g., Doors and Windows). Further, Spaces can contain Installations and Furniture, i.e., fixed and movable objects.

Geometry Model: Our geometry model is based on the Geography Markup Language 3 (GML) [15], which is capable of describing 3D geometry and also serves as a basis for CityGML. For each BuildingObject and for each LOD, an appropriate geometric representation is specified, i.e., 2D geometries for LOD-1 and LOD-2, and 3D geometries for LOD-3 and LOD-4.

Routing Model: Our routing model defines ConnectionPoints and Connections. Their semantics is introduced indirectly by referencing BuildingObjects such as stairways and elevators. A ConnectionPoint is either located inside a Space or outside of a Floor. The latter one allows for connecting to outdoor navigation networks such as the public transportation system.

For ConnectionPoints and Connections, a geometric representation, e.g., for intermediate way points, can be provided. Connections between different spaces are specified explicitly, those within a single space could be left unspecified. In this case, the route finding service has to determine the intra-space routing network. 


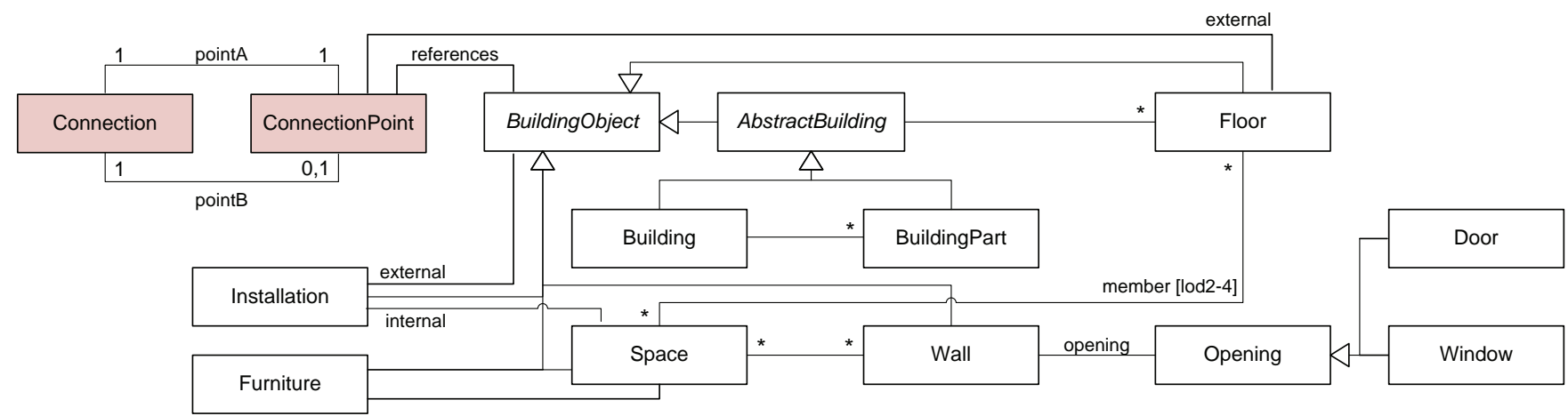

Figure 2. Thematic and routing model of our proposed indoor LOD model.

The distinction between Connections an respective ConnectionPoints enables a complex modeling of routing information. This can include door access restrictions or the waiting time in front of a elevator.

Fig. 3 illustrates an instance of our routing model that contains internal and external ConnectionPoints, as well as bidirectional and unidirectional Connections, which reference doors and windows. The small window on the right is not traversable, and the elevator's ConnectionPoint would be linked explicitly to the one in the floor above.

Appearance Binding: For interoperable representations of indoor routing data, an appearance specification is required that enables the adjustment of visual parameters, such as colors, materials, or textures, by surface and line styles. Installations and Furniture, e.g., fire extinguishers, can be represented by specific glyphs or icons. This can be supported by using the surface-oriented appearance model of CityGML and the symbolizer concept of the Symbology Encoding (SE) standard [14] [12].

Domain-Specific Extensions: Extendibility is a main requirement of an interoperable indoor model. Thus, we suggest an extension model as provided by CityGML: Additional thematic types can be derived from BuildingObject or subtypes, and properties and attributes can be added to existing types.

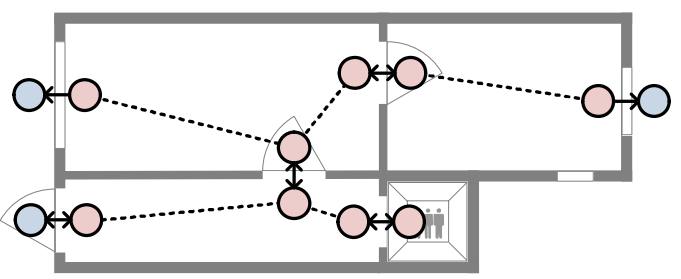

Figure 3. Example of LOD-2 building and routing model instances for a single floor.

\subsection{Level-of-Detail Mapping}

Tab. 1 lists the characteristics of the proposed indoor LOD model visualized in Fig. 1 and suggests model scales and geometric accuracies. In the following, for each LOD the indoor model is explained. The mapping to GML geometries is described in Tab. 2.

LOD-1: The thematic LOD-1 model contains only Buildings and BuildingParts which can be described by the 2D geometry of their Floors. The LOD-1 routing model is limited to external ConnectionPoints, which allow for outdoor connectivity (e.g., streets, footpaths, shuttle service, or public transportation system). Thereby, LOD-1 representations can serve as interfaces to outdoor routing and visualization models. If using multiple floor layers in visualization, this LOD can help to avoid visual clutter as they do not contain detailed entities such as rooms or furniture.

LOD-2: In LOD-2 the thematic model is enriched by Spaces, Walls, and Openings, which can be geometrically represented by 2D MultiCurves. Additionally, relevant Installations and Furniture can be integrated - either as 2D Point, or 2D MultiSurface that represents a 2D prototype shape. For representation purpose, their appearance specification could map these geometries to glyph depictions. This supports simple labeling techniques and the application of $2 \mathrm{D}$ routes to a respective visualization. Compared to LOD-1, the LOD-2 routing graph is extended by internal ConnectionPoints, which can reference entities of the thematic model.

LOD-3: LOD-3 represents a 3D parameterization of the LOD-2 model. It is mainly motivated by different requirements for $2 \mathrm{D}$ and $3 \mathrm{D}$ representations, which are, e.g., geometric complexity, graphics formats, rendering hardware, interaction capabilities, and display properties. In LOD-3, BuildingObjects and routing entities are linked 
Table 1. Characteristics of proposed indoor LOD model for route visualization.

\begin{tabular}{|c|c|c|c|c|}
\hline Characteristics & LOD-1 & LOD-2 & LOD-3 & LOD-4 \\
\hline Model scale description & 2D floor footprints & 2D floor plans & $\begin{array}{l}\text { 3D floor plans and 3D } \\
\text { selectional views }\end{array}$ & $\begin{array}{l}\text { high detailed } \\
3 \mathrm{D} \text { rooms }\end{array}$ \\
\hline $\begin{array}{l}\text { Geometric accuracy } \\
\text { (position[/height]) }\end{array}$ & $\begin{array}{l}\text { low } \\
(0.5 \mathrm{~m})\end{array}$ & $\begin{array}{l}\text { middle } \\
(0.2 \mathrm{~m})\end{array}$ & $\begin{array}{l}\text { middle } \\
(0.2 / 0.2 \mathrm{~m})\end{array}$ & $\begin{array}{l}\text { high } \\
(0.05 / 0.05 \mathrm{~m})\end{array}$ \\
\hline Route visualization & $\begin{array}{l}\text { only external } \\
\text { connection points }\end{array}$ & $\begin{array}{l}\text { 2D routes at the } \\
\text { Space-level }\end{array}$ & $\begin{array}{l}\text { 3D routes at the } \\
\text { Space-level }\end{array}$ & $\begin{array}{l}\text { 3D routes at the } \\
\text { Installation- and } \\
\text { Furniture-level }\end{array}$ \\
\hline
\end{tabular}

Table 2. Mapping GML geometries to thematic types in the various LODs.

\begin{tabular}{lllll}
\hline Thematic type & LOD-1 & LOD-2 & LOD-3 & LOD-4 \\
\hline Floor & 2D MultiSurface & 2D MultiSurface & 3D MultiSolid & 3D MultiSolid \\
\hline Space & - & 2D MultiSurface & 3D MultiSurface & $\begin{array}{l}\text { 3D MultiSurface } \\
\text { 3D MultiSolid }\end{array}$ \\
\hline Wall & - & 2D MultiCurve & 3D MultiSurface & 3D MultiSurface \\
& & & 3D MultiSolid & 3D MultiSurface \\
\hline Opening & - & 2D MultiCurve & 3D MultiSurface & 3D MultiSolid \\
\hline Furniture/ & - & 3D MultiSolid & 3D MultiSurface \\
Installation & & 2D MultiSurface & 3D Point & 3D MultiSolid \\
\hline ConnectionPoint & 2D Point (externals) & 2D Point & 3D MultiSolid & 3D Point \\
\hline Connection & - & 2D MultiCurve & 3D MultiCurve & 3D MultiCurve \\
\hline
\end{tabular}

to 3D geometries. Relevant Installations and Furniture are represented by 3D locations or 3D prototype shapes. Depictions of items usable as landmarks can be integrated in the visual representation.

LOD-4: LOD-4 provides the most detailed space information, the highest geometric accuracy, and most detailed representation. As main characteristic, their geometry represents not only prototypes but their real forms. Fig. 1 shows a section of such a model. Representations of such complexity are currently hand-made by designers or architects using 3D CAD systems.

\section{Conclusions \& Future Work}

This paper introduces and discusses a novel concept for an indoor LOD classification and model, which can be applied to applications and systems that provide indoor route planning and visualization. The LOD model covers different information dimensions of indoor models including thematic, geometric, and routing-related information and helps to design solutions that can cope with massive indoor models. The LOD schema has been designed in close analogy to existing standards such as CityGML and IFC to support automatic data extraction, to facilitate adoption, and to take advantage of LOD concepts for virtual 3D city models.

A potential limitation of our LOD approach is, that we assume that a complex building can be decomposed into separate floors, which generally is not possible for all types of buildings. In addition, our model off-loads a considerable problem to the routing component implementing the model, as it needs to resolve implicit graph edges in addition to graph computations, which may get complex in the case of 3D spaces. It is also still unclear, how especially high detail LOD-4 models will be modeled and maintained. However, using tracking technologies such as RFID could reduce the manual effort to a minimum and, e.g., libraries could perfectly benefit from precise location and routing.

In the future we plan to refine this first proposal with more detailed information and evaluate it by a prototypic implementation. Especially, a detailed appearance model is needed for flexible visualization. As our main focus is routing visualization, we plan to implement advanced routing visualization using focus \& context techniques, e.g., by combining different LOD representations based on regions of interest. In the long term, we focus on automatic derivation of LODs based on standardized exchange formats for geospatial data, such as CityGML, IFC or other BIM related formats. We will further analyze common and standardized appearances and visualization rendering styles. 


\section{Acknowledgments}

This work has been partly funded by the German Federal Ministry of Education and Research (BMBF) as part of the InnoProfile research group 3D Geoinformation (www.3dgi.de). We thank Christoph Neijenhuis for provided data. We also thank the anonymous reviewers for valuable comments.

\section{References}

[1] T. H. Kolbe, G. Gröger, and L. Plümer, "CityGML - Interoperable Access to 3D City Models," in Proc. of 1st Int. Symp. on Geoinformation for Disaster Management. Springer, 2005.

[2] Industry Foundation Classes - IFC2x Edt. 3, International Alliance for Interoperability, 2007, http://www.buildingsmart.com/bim (10.03.2009). [Online]. Available: http://www.buildingsmart.com/bim

[3] D. Luebke, M. Reddy, J. D. Cohen, A. Varshney, B. Watson, and R. Huebner, Level of Detail for 3D Graphics. New York, NY, USA: Elsevier Science Inc., 2002.

[4] R. Sedgewick and C. J. V. Wyk, Algorithms in C++: Fundamentals, Data Structures, Sorting, Searching. AddisonWesley Longman Publishing Co., Inc., 1998.

[5] B. Liu, "Intelligent Route Finding: Combining Knowledge, Cases and an Efficient Search Algorithm," in Proc. of 12th Europ. Conf. on Artificial Intelligence. Wiley \& Sons, Ltd, 1996, pp. 380-384.

[6] V. Tsetsos, C. Anagnostopoulos, P. Kikiras, T. Hasiotis, and S. Hadjiefthymiades, "A Human-centered Semantic Navigation System for Indoor Environments," in Int. Conf. on Pervasive Services ICPS '05, 2005, pp. 146-155.

[7] H. Hu and D.-L. Lee, "Semantic Location Modeling for Location Navigation in Mobile Environment," in Proc. of IEEE MDM, 2004, pp. 52-61.

[8] B. Lorenz and H. J. Ohlbach, "A Hybrid Spatial Model for Representing Indoor Environments," in Proc. of 6th W2GIS. Springer, 2006.

[9] E.-P. Stoffel, K. Schoder, and H. J. Ohlbach, "Applying Hierarchical Graphs to Pedestrian Indoor Navigation," in Proc. of 16th ACMGIS. ACM, 2008.
[10] J. Lee, "3D GIS for Geo-coding Human Activity in Microscale Urban Environments," in Lecture Notes in Computer Science, 2004

[11] T. Becker, C. Nagel, and T. H. Kolbe, "A Multilayered SpaceEvent Model for Navigation in Indoor Spaces," in Proc. of 3rd 3D-GeoInfo. Springer, 2008.

[12] Y. Li and Z. He, "3D Indoor Navigation: A Framework of Combining BIM with 3D GIS," in Proc. of 44th ISOCARP Congress, 2008.

[13] F. Shayeganfar, A. Anjomshoaa, and A. M. Tjoa, "A Smart Indoor Navigation Solution Based on Building Information Model and Google Android," in ICCHP. Springer, 2008, pp. 1050-1056.

[14] B. Brunner-Friedrich and V. Radoczky, "Active Landmarks in Indoor Environments," in Proc. of VISUAL 2005. Springer, 2005.

[15] S. Mohan, A. Zipf, and A. Weiser, "Supporting Indoor Evacuation through Landmarks on Mobile Displays," in Joined CIG/ISPRS Conf. on Geomatics for Disaster and Risk Management, 2007.

[16] F. Zhang and H. Sun, "Dynamic Labeling Management in Virtual and Augmented Environments," in Proc. of CAD-CG '05. IEEE Computer Society, 2005, pp. 397-402.

[17] C. Niederauer, M. Houston, M. Agrawala, and G. Humphreys, "Non-Invasive Interactive Visualization of Dynamic Architectural Environments," ACM Transactions on Graphics, vol. 22 , no. 3, p. 700, 2003.

[18] X. Yin, P. Wonka, and A. Razdan, "Generating 3D Building Models from Architectural Drawings: A Survey," IEEE Computer Graphics and Applications, vol. 29, no. 1, pp. 20-30, 2009.

[19] OpenGIS Geography Markup Language (GML) Encoding Standard, Version 3.2.1, OpenGIS Implementation Specification, Open Geospatial Consortium Inc., August 2007.

[20] Symbology Encoding Implementation Specification, Version 1.1.0, OpenGIS Implementation Specification, Open Geospatial Consortium Inc., July 2006.

[21] S. Neubauer and A. Zipf, "Suggestions for Extending the OGC Styled Layer Descriptor (SLD) Specification into the Third Dimension - An Analysis of Possible Visualization Rules for 3D City Models," in Urban Data Management Symposium, Stuttgart, Germany, October 2007. 